\title{
Four-dimensional traversable wormholes and bouncing cosmologies in vacuum
}

\author{
Andrés Anabalón ${ }^{a, b}$ and Julio Oliva ${ }^{c}$ \\ ${ }^{a}$ Departamento de Ciencias, Facultad de Artes Liberales, \\ Universidad Adolfo Ibáñez, Avenida Padre Hurtado 750, Viña del Mar, Chile \\ ${ }^{b}$ Max-Planck-Institut für Gravitationsphysik (Albert-Einstein-Institut), \\ Am Mühlenberg 1, D-14476 Potsdam, Germany \\ ${ }^{c}$ Departamento de Física, Universidad de Concepción, \\ Casilla 160-C, Concepción, Chile \\ E-mail: andres.anabalon@uai.cl, juoliva@udec.cl
}

ABSTRACT: In this letter we point out the existence of solutions to General Relativity with a negative cosmological constant in four dimensions, which contain solitons as well as traversable wormholes. The latter connect two asymptotically locally $\mathrm{AdS}_{4}$ spacetimes. At every constant value of the radial coordinate the spacetime is a spacelike warped $\mathrm{AdS}_{3}$. We compute the dual energy momentum tensor at each boundary showing that it yields different results. We also show that these vacuum wormholes can have more than one throat and that they are indeed traversable by computing the time it takes for a light signal to go from one boundary to the other, as seen by a geodesic observer. We generalize the wormholes to include rotation and charge. When the cosmological constant is positive we find a cosmology that is everywhere regular, has either one or two bounces and that for late and early times matches the Friedmann-Lemaitre-Robertson-Walker metric with spherical topology and an exponential scale factor.

KeYwords: AdS-CFT Correspondence, Classical Theories of Gravity

ARXIV EPRINT: 1811.03497 


\section{Contents}

1 Introduction 1

2 The spacelike warped $\mathrm{AdS}_{3} \quad 2$

3 Wormhole-like slicing of $\mathrm{AdS}_{4} \quad 3$

4 The wormhole solution 3

5 Absence of closed timelike curves 5

$\begin{array}{llr}6 & \text { Holographic renormalization } & 6\end{array}$

$\begin{array}{llr}7 & \text { Holographic stability } & 6\end{array}$

8 The charged and spinning generalization $\quad 7$

9 Bouncing cosmologies $\quad 8$

$\begin{array}{lr}10 \text { Discussion } & 8\end{array}$

\section{Introduction}

Wormholes have a long and rich history in physics and the pop culture. The idea was originally introduced by Einstein and Rosen [1]. The motivation was the description of particles as gravitational and electromagnetic fields without singularities. Nowadays we understand that the Einstein-Rosen bridge is just a part of the Kruskal extension of the Schwarzschild metric and that the non-singular bridge to other universe is non-travesable.

Traversable wormholes are spacetimes that causally connect two far away regions by means of a throat. Despite the general interest in their existence, they are still not part of an accepted piece of knowledge within theoretical physics. This situation should change with this letter.

Indeed, a major open problem in physics is whether wormholes can take place in physically sensible and simple circumstances. It is well-established that asymptotically flat gravity in four dimensions requires exotic matter fields or to go beyond General Relativity to produce a wormhole [2, 3]. Holography [4], uses asymptotically AdS gravity to describe a conformal field theory (and its deformations). In this context, a careful study shows that Einstein wormholes with a boundary of positive curvature do not not exist [5]. From the holographic point of view it is problematic to define a field theory on a manifold of negative curvature. This is because the scalar fields in the dual field theory are conformally coupled to the scalar curvature. Therefore, they have an effective negative squared mass, which would spoil the stability of the system. We circunvent this dilemma by using AdS 
itself as the boundary of the wormhole. Indeed, in AdS, there are tachyonic masses that do not introduce instabilities and the squared mass of the conformally coupled scalar fields, although negative, is always safe in this regard [6, 7]. When picking the $\mathrm{AdS}_{3}$ boundary it is easy to see that the bulk solution presented below is a smooth deformation in such a way that the surfaces of constant radial coordinate are spacelike warped AdS [8]. Therefore, it is also natural to consider wormholes where the boundary itself is warped. This is exactly what is done in this paper.

Another major open problem in physics is to find a non-singular description of the Big-Bang. A realization of this idea is known as bouncing cosmologies, which have been shown to be compatible with cosmological data and a viable alternative for inflation [9]. However, before this article, no simple example of a bouncing cosmology was known with no ad-hoc matter fields or exotic kinetic terms [10]. We use only the Einstein equations and a positive cosmological constant. The crucial step to construct this long sought spacetime is to allow for space anisotropies at the bounce. Notwithstanding these anisotropies we show that, by an adequate election of the parameters in the metric, the late evolution of the spacetime can be chosen to be exactly the everywhere homogeneous and isotropic de Sitter spacetime. Thus, our bouncing cosmologies provide a new arena to explore the cosmology of our Universe without the problem of the initial singularity.

The mathematics involved in our construction are fairly simple. We review some of the most interesting geometrical ingredients in the first two sections. First, we show how to deform $\mathrm{AdS}_{3}$ in a smooth way and without introducing closed timelike curves [8]. As we shall see our Einstein wormhole is exactly a spacelike warped AdS spacetime at every constant value of the radial coordinate. The boundary can be warped or not, depending on an integration constant that controls the warping at infinity. Later we discuss how the slicing of $\mathrm{AdS}_{4}$ by $\mathrm{AdS}_{3}$ superficially resembles a wormhole. However, the existence of a globally defined change of coordinates from the $\mathrm{AdS}_{3}$ slicing to the sphere slicing can be used to proof that global $\mathrm{AdS}_{4}$ has a single boundary. Then we propose an ansatz to construct a spacetime with a (warped) $\mathrm{AdS}_{3}$ boundary. The wormhole spacetime arises thus naturally. It is then shown that it can have either a single or two throats and an anti-throat. The time that takes a photon to go from one boundary to the other is computed. We then give an elegant argument on the absence of closed time like curves on the spacetime. We compute the dual energy momentum tensor at each boundary, yielding different results. Later we study the hologrophic stability of the system. We show that a conformally coupled scalar field of the putative CFT have perfectly well defined dynamics and the unstability of negatively curved boundaries considered in [5] does not arise in our case. The wormhole solution is then embedded in a general class of metrics that contains all Einstein black hole solutions in four dimensions. This allow us to obtain its charged and spinning form. Finally, we make analogous considerations when the cosmological constant is positive, obtaining a bouncing cosmology that can be de Sitter at late or early times.

\section{The spacelike warped $\mathrm{AdS}_{3}$}

$\mathrm{AdS}_{3}$ with radius $\lambda$ can be written as

$$
d s_{A d S_{3}}^{2}=\frac{\lambda^{2}}{4}\left[-\cosh ^{2} \theta d t^{2}+d \theta^{2}+(d u+\sinh (\theta) d t)^{2}\right],
$$


where the coordinates satisfy $(t, \theta, u) \in \mathbb{R}^{3}$. The isometry of $\mathrm{AdS}_{3}, \mathrm{SO}(2,2)$, is broken to $\mathrm{SL}(2, \mathbb{R}) \times \mathbb{R}=\mathrm{GL}(2, \mathbb{R})$ in the warped metric:

$$
d s_{W A d S_{3}}^{2}=\gamma_{i j} d x^{i} d x^{j}=\frac{\lambda^{2}}{\nu^{2}+3}\left[-\cosh ^{2} \theta d t^{2}+d \theta^{2}+\frac{4 \nu^{2}}{\nu^{2}+3}(d u+\sinh (\theta) d t)^{2}\right] .
$$

The spacetime (2.2) is a smooth manifold, free of closed timelike curves. It is a Lorentzian version of the squashed three pseudosphere. Spacelike warped $\mathrm{AdS}_{3}$ [8], and their black holes [11], have been extensively studied and they arise as solutions of topologically massive gravity with graviton mass $\mu=\frac{3 \nu}{\lambda}$. There is also a pathological version of (2.2), known as timelike warped $\mathrm{AdS}_{3}$ which does contain closed timelike curves. In this paper, we shall only focus on the physically relevant case (2.2).

\section{Wormhole-like slicing of $\mathrm{AdS}_{4}$}

As is well known, it is possible to slice $\mathrm{AdS}_{4}$ in $\mathrm{AdS}_{3}$ submanifolds as follows

$$
g_{\alpha \beta} d x^{\alpha} d x^{\beta}=\frac{\ell^{2} d r^{2}}{r^{2}+1}+\frac{\ell^{2}}{4}\left(r^{2}+1\right)\left[-\cosh (\theta)^{2} d t^{2}+d \theta^{2}+(d u+\sinh (\theta) d t)^{2}\right]
$$

where $\ell$ is the $A d S_{4}$ radius, i.e.

$$
R_{\alpha \beta}=-\frac{3}{\ell^{2}} g_{\alpha \beta}
$$

for the metric (3.1). While this slicing seems to have a wormhole throat at $r=0$ and two disconnected boundaries at $r= \pm \infty$, this is just an artifact of the coordinates. There is a well-known global change of coordinates that maps (3.1) to standard global AdS with a round sphere at the boundary.

The fact that the two boundaries of (3.1) are connected has been remarked in [12], where by performing identifications in the fixed- $r$ manifold it was pointed out that is possible to disconnect the two boundaries at $r= \pm \infty$. This is simply because the change of coordinates that maps (3.1) to global AdS stop existing as a by-product of the identification. In the next section we show how is possible to have a wormhole by resorting to geometry instead of topology.

\section{The wormhole solution}

It is our interest to obtain (2.2) as the boundary of an asymptotically $\mathrm{AdS}_{4}$ Einstein space. Hence, it is natural to propose the following ansatz

$$
d s^{2}=\frac{4 \ell^{4} d r^{2}}{\sigma^{2} f(r)}+g(r)\left(-\cosh ^{2}(\theta) d t^{2}+d \theta^{2}\right)+f(r)(d u+\sinh (\theta) d t)^{2} .
$$

The Einstein equations (3.2) are satisfied provided

$$
\begin{aligned}
& g(r)=\frac{\ell^{2}}{\sigma}\left(r^{2}+1\right), \\
& f(r)=\frac{4 \ell^{2}}{\sigma^{2}} \frac{r^{4}+(6-\sigma) r^{2}+\ell m r+\sigma-3}{r^{2}+1},
\end{aligned}
$$




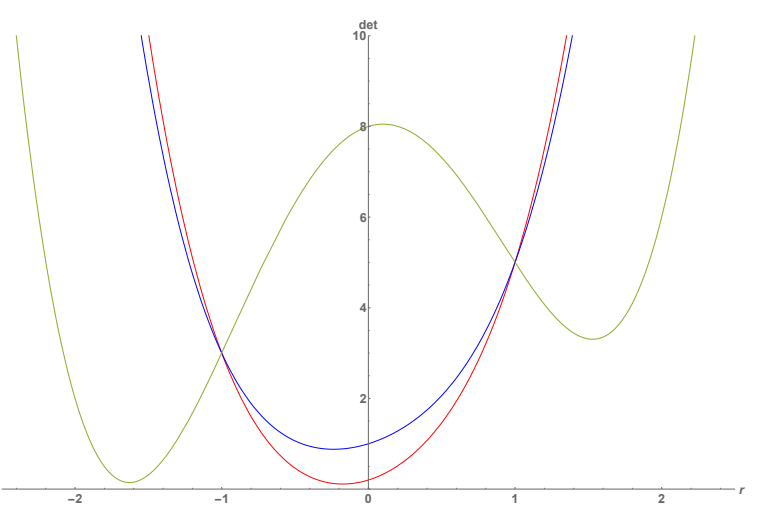

Figure 1. Here we plot the dimensionless determinant of the spatial sections with constant, $(t, r)$ det $=f(r) g(r) \sigma^{3} \ell^{-4} / 4$ versus the $r$ coordinate. All the plots have $m \ell=1$. The plots are for $\sigma=3.2$ and $\sigma=4$ that have a single throat (from down up) and $\sigma=11$ with two throats and an anti-throat. As expected, det is asymmetric unless $m=0$. For a given $m$ all curves intersect at $r= \pm 1$ as $f$ is independent of $\sigma$ there.

Where $\sigma$ and $m$ are integration constants. We are interested in the case where $f$ has no real zero. An straightforward analysis shows that $f$ never vanishes provided

$$
12>\sigma>3, \quad|\ell m|<\frac{2}{3 \sqrt{3}}(12-\sigma) \sqrt{\sigma-3} .
$$

Thus, for these ranges of the parameters, the metric functions are everywhere positive and regular and the range of the $r$-coordinate is

$$
\infty>r>-\infty
$$

The Kretschmann invariant is

$$
\begin{aligned}
R^{\mu \nu \alpha \beta} R_{\mu \nu \alpha \beta}= & \frac{24}{\ell^{4}}-\frac{12\left(r^{2}-1\right)\left(\left(r^{2}+1\right)^{2}-16 r^{2}\right)\left[4(\sigma-4)^{2}-m^{2} \ell^{2}\right]}{\ell^{4}\left(r^{2}+1\right)^{6}} \\
& +\frac{96(\sigma-4) r m\left(r^{2}-3\right)\left(3 r^{2}-1\right)}{\ell^{3}\left(r^{2}+1\right)^{6}} .
\end{aligned}
$$

It is possible to see that for $\sigma=4$ and $m=0$, the spacetime is everywhere constant curvature and coincides with (3.1). The interpretation of (2.1) as a wormhole is now straightforward.

As shown by figure 1, the wormhole goes from having a single throat for $\sigma \leq 6$ to have two throats for $\sigma>6$. The two throats must have a local maximum in between that we call an anti-throat.

The scaled timelike coordinate $\ell t / \sigma^{1 / 2}$ coincides with the proper time of a geodesic observer located at $r=0=\theta$. According to this observer, the time it takes for a light ray to go from one boundary to the other is finite, which is expected since the spacetime is asymptotically $\mathrm{AdS}_{4}$ at both asymptotic regions. The crossing time is given by

$$
\Delta t=\frac{2 \ell^{2}}{\sigma} \int_{-\infty}^{+\infty} \frac{d r}{\sqrt{f(r) g(r)}} .
$$




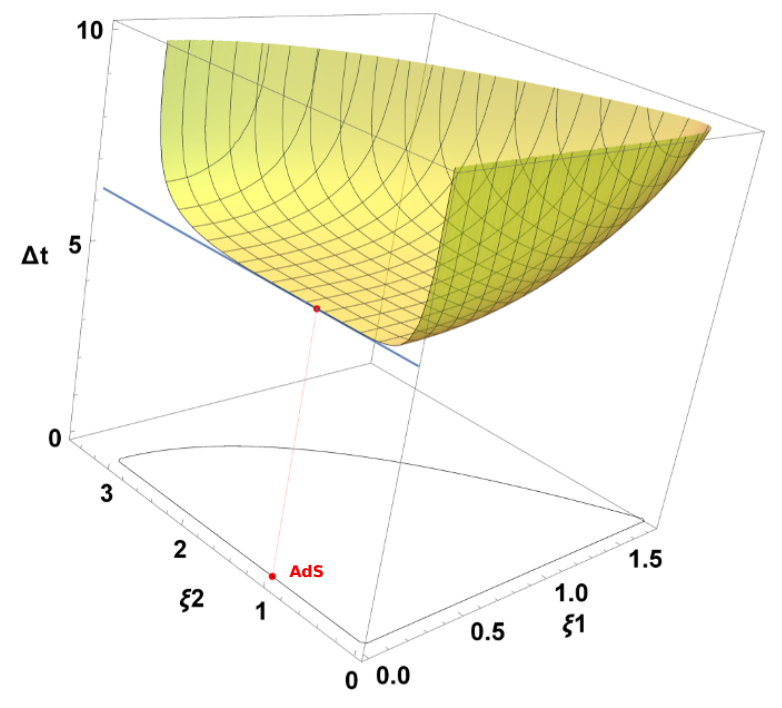

Figure 2. Crossing time $\Delta t$ as a function of the parameters $0<\xi_{1}<\sqrt{3}$ and $0<\xi_{2}<3-\xi_{1}^{2}$. The red dot corresponds to $m=0$ and $\sigma=4$, i.e. global $\mathrm{AdS}_{4}$ which does not represent a wormhole. $\Delta t$ has a global minimum at that point $\left(\Delta t_{\min }=2 \pi\right)$. Outside of the AdS point the metric has two asymptotic regions and $\Delta t$ is the time it takes to a photon to go from one asymptotic region to the other, as seen by a geodesic observer at $r=0=\theta$. As suggested in the plot, if one approaches the boundaries of the domain on the plot $\left(\xi_{1}, \xi_{2}\right), \Delta t$ grows unboundedly.

To make this integral, and plot it, it is conveniente to write the metric function $f(r)$ in term of its roots, namely

$$
f(r)=\frac{4 \ell^{2}}{\sigma^{2}} \frac{\left(r-z_{1}\right)\left(r-z_{1}^{*}\right)\left(r-z_{2}\right)\left(r-z_{2}^{*}\right)}{r^{2}+1},
$$

with $\sigma=2 \xi_{1}^{2}+6-\xi_{2}-\zeta, z_{1}=\xi_{1}+I \sqrt{\xi_{2}}, z_{2}=-\xi_{1}+I \sqrt{\zeta}$ and $\zeta=\frac{\left(\xi_{1}^{2}+1\right)\left(3-\xi_{1}^{2}-\xi_{2}\right)}{1+\xi_{1}^{2}+\xi_{2}} . f(r)$ has no real zero provided $\xi_{2}>0$ and $0<\xi_{1}^{2}<3-\xi_{2}$. This is the region we have plotted. Figure 2 depicts the crossing time as a function of the parameters $\left(\xi_{1}, \xi_{2}\right)$.

\section{Absence of closed timelike curves}

This argument is a slight generalization of the one in [13]. We note that the coordinates $(t, u, \theta, r)$ in (4.1) provide a global covering of the manifold. A closed timelike curve satisfies

$$
\begin{aligned}
0<\left(g(r) \cosh (\theta)^{2}-f(r)\right. & \left.\sinh (\theta)^{2}\right)\left(\frac{d t}{d \tau}\right)^{2}-2 f(r) \sinh (\theta) \frac{d t}{d \tau} \frac{d u}{d \tau} \\
& -f(r)\left(\frac{d u}{d \tau}\right)^{2}-g(r)\left(\frac{d \theta}{d \tau}\right)^{2}-\frac{1}{f(r)}\left(\frac{d r}{d \tau}\right)^{2}
\end{aligned}
$$

where $\tau$ yields a good parametrization of the curve. If the curve is closed, $t$ must come back to its original value. Hence, there must be a point where $\frac{d t}{d \tau}=0$. Taking into account that $f$ and $g$ are everywhere positive functions, it is straightforward to see that $\frac{d t}{d \tau}=0$ is in contradiction with (5.1). 


\section{Holographic renormalization}

We now pass to find what is the dual energy momentum tensor associated to this spacetime. The procedure is as follows. The action, including boundary counterterms [14, 15], is

$$
I[g]=\frac{1}{2 \kappa} \int_{M} d^{4} x \sqrt{-g}\left[R+\frac{6}{\ell^{2}}\right]+\frac{1}{\kappa} \int_{\partial M} d^{3} x \sqrt{-h} \mathcal{K}-\frac{1}{\kappa} \int_{\partial M} d^{3} x \sqrt{-h}\left[\frac{2}{\ell}+\frac{\ell}{2} \mathcal{R}(h)\right],
$$

where $\kappa=8 \pi G, \mathcal{K}_{\mu \nu}$ is the extrinsic curvature of the boundary metric, $h_{\mu \nu}=g_{\mu \nu}-N_{\mu} N_{\nu}$ is the induced metric on the fixed $r$ hypersurfaces and $\mathcal{R}$ its Ricci curvature. $N_{\mu}=\delta_{\mu}^{r} \sqrt{g_{r r}}$ is the outward pointing normal, where we assume that $r>0$, the case with $r<0$ will be discussed below. Varying the action gives the energy momentum tensor:

$$
\kappa \mathcal{T}_{\mu \nu}=\ell \mathcal{G}_{\mu \nu}(h)-\frac{2}{\ell} h_{\mu \nu}-\mathcal{K}_{\mu \nu}+h_{\mu \nu} \mathcal{K} .
$$

The boundary metric is $\gamma_{i j}=\lim _{r \rightarrow \infty} \frac{1}{r^{2}} h_{i j}$, and is given by (2.2) with $\frac{\lambda^{2}}{\nu^{2}+3}=\frac{\ell^{2}}{\sigma}$ and $\nu^{2}=\frac{3}{\sigma-1}$. The dual enery momentum tensor is

$$
\left\langle\mathcal{T}_{i j}^{+}\right\rangle=\lim _{r \longrightarrow \infty} r \mathcal{T}_{i j}
$$

which yields

$$
\left\langle\mathcal{T}_{i j}^{+}\right\rangle d x^{i} d x^{j}=\frac{\ell^{2}}{\sigma \kappa}\left[-\frac{m}{2}\left(-\cosh ^{2}(\theta) d t^{2}+d \theta^{2}\right)+\frac{4 m}{\sigma}(d u+\sinh (\theta) d t)^{2}\right] .
$$

The wormhole spacetime is invariant under the combined changes $r \rightarrow-r$ and $m \rightarrow-m$. Hence, the energy-momentum tensor for $r<0$ is the same than $\mathcal{T}_{i j}^{+}$changing $m \rightarrow-m$. We note that the factor in front of the energy momentum tensor can be translated to field theory variables $\frac{\ell^{2}}{\kappa}=\frac{2^{1 / 2}}{12 \pi} k^{1 / 2} N^{3 / 2}$ where we have used the standard holographic dictionary to identify $k$ with the level and $N$ with the rank of the gauge groups of the ABJ(M) theory, see for instance [16].

The bulk solution is everywhere regular. Therefore, the dual energy momentum tensor is continuous along the boundary of the spacetime. The fact that each boundary has a different energy momentum tensor is a physicist's proof that the boundary is not connected.

\section{Holographic stability}

Let us now deal with the issue of the stability of the CFT on the boundary metric $\gamma$. The first thing one would like to check is what happens with a simple conformally coupled scalar field in three dimensions when propagating on this geometry. All objects in this section are defined in terms of the metric $\gamma$. The scalar field equation is

$$
\square \phi-\frac{1}{8} R[\gamma] \phi=0 \text {. }
$$

The following ansatz separates the field equation

$$
\phi=\cos (k u+\omega t) H(\theta) .
$$


The leading order of the field equation (7.1) for large values of $\theta$ is

$$
4 \sigma\left(\frac{d^{2} H}{d \theta^{2}}+\frac{d H}{d \theta}\right)+\left(\sigma-1-\sigma^{2} k^{2}+4 \sigma k^{2}\right) H=0 .
$$

The asymptotic form of $H$ is then

$$
H=\phi_{+} e^{\Delta_{+} \frac{\theta}{\sqrt{\sigma}}}+\phi_{-} e^{\Delta_{-} \frac{\theta}{\sqrt{\sigma}}}+\ldots
$$

where

$$
\Delta_{ \pm}=\frac{-\sigma \pm \sqrt{\sigma+k^{2} \sigma^{2}(\sigma-4)}}{2 \sqrt{\sigma}} .
$$

It can be readily noticed that for $\sigma<4$ and large enough $k$ the scalar field will always develop an unstability due to the complex $\Delta_{ \pm}$. For $\sigma \geq 4$, the wormholes are stable under scalar field fluctuations of the CFT. More details of the stability of warped $\mathrm{AdS}_{3}$ can be found in [17].

\section{The charged and spinning generalization}

So far we have studied the simplest case where the wormhole is static. It is natural to generalize the spacetime to introduce charge and spin. An educated guess lead us to consider the Plebanski-Demianski [18] family of spacetimes in four dimensions

$$
\begin{aligned}
d s^{2}=\frac{1}{(q-A p)^{2}}\left[-\frac{X(p)}{1+\xi^{2} q^{2} p^{2}}\left(d \tau-\xi q^{2} d \phi\right)^{2}\right. & +\frac{Y(q)}{1+\xi^{2} q^{2} p^{2}}\left(d \phi+\xi p^{2} d \tau\right)^{2} \\
& \left.+\left(1+\xi^{2} q^{2} p^{2}\right)\left(\frac{d q^{2}}{Y(q)}+\frac{d p^{2}}{X(p)}\right)\right]
\end{aligned}
$$

with the gauge field

$$
B=p \frac{Q+P \xi p q}{1+\xi^{2} q^{2} p^{2}} d \tau+q \frac{P-Q \xi p q}{1+\xi^{2} q^{2} p^{2}} d \phi .
$$

The Einstein-Maxwell equations

$$
R_{\mu \nu}-\frac{1}{2} g_{\mu \nu} R-\frac{3}{\ell^{2}} g_{\mu \nu}=2 \kappa\left(F_{\mu \sigma} F_{\nu}^{\cdot \sigma}-\frac{1}{4} g_{\mu \nu} F_{\alpha \beta} F^{\alpha \beta}\right), \quad \nabla^{\mu} F_{\mu \nu}=0,
$$

with $F_{\mu \nu}=\partial_{\mu} B_{\nu}-\partial_{\nu} B_{\mu}$, are satisfied provided

$$
\begin{aligned}
& Y=\ell^{-2}-A^{2} \xi^{-2}\left(Q_{T}^{2}+y_{4}\right)-y_{1} q A+y_{2} q^{2}+y_{3} q^{3}+y_{4} q^{4}, \\
& X=\xi^{-2}\left(Q_{T}^{2}+y_{4}\right)+y_{1} p-y_{2} p^{2}-A y_{3} p^{3}+\left(\xi^{2} \ell^{-2}-A^{2} y_{4}\right) p^{4},
\end{aligned}
$$

where $Q_{T}^{2}=\kappa Q^{2}+\kappa P^{2}$. This solution is known to contain all spinning black holes in four dimensions as special limits. To retrieve the wormhole we found that is necessary to set the acceleration parameter to zero, $A=0$, make the following change of coordinates

$$
\begin{aligned}
\tau(t, u) & =-\frac{\ell^{2}}{n^{2} \sigma}(t+2 \epsilon \alpha u), & \phi(t, u) & =\frac{\ell^{2}}{n \sigma}\left(4 u+\frac{t}{\alpha \epsilon}\right), \\
q(r) & =\frac{1}{r n}, & p(y) & =y+\frac{1}{\epsilon \alpha}
\end{aligned}
$$


and the reparemeterization

$$
\begin{array}{ll}
y_{2}=\ell^{-2} n^{2}\left(6+\epsilon \sigma+\alpha^{2} \epsilon\right), & y_{4}=-\ell^{-2} n^{2}\left(3-\alpha^{2} \epsilon^{2} \sigma-3 \alpha^{2} \epsilon+\sigma \epsilon\right)-Q_{T}^{2}, \\
y_{1}=-2 n^{2} \ell^{-2} \frac{\left(-4+\alpha^{2} \epsilon-\sigma \epsilon\right)}{\epsilon \alpha}, \quad \xi=\epsilon n \alpha, \quad y_{3}=\ell^{-1} m n^{3} .
\end{array}
$$

Then the metric and the gauge field have a smooth $\alpha=0$ limit which exactly coincides with the wormhole with $y=\sinh (\theta)$ (4.1) provided $\epsilon=-1$ and $Q_{T}=0$. When $Q_{T} \neq 0$ the static wormhole is charged and the $\alpha=0$ limit is well defined provided a singular gauge transformation is substracted from the gauge field. $\epsilon$ controls the topology of the boundary. For $\epsilon=0$ the boundary has no curvature and when $\epsilon=1$ the curvature is positive. It can be seen that there are wormholes only when $\epsilon=-1$, otherwise the spacetime describe AdS solitons.

If $\alpha \neq 0$ then the interpretation of the spacetime as a wormhole is less simple. The metric is singular at $r=0$ and $y=-1$. At every constant radial coordinate there is a black hole, which is regular at the $r= \pm \infty$ boundaries. The black hole flows into the bulk through the $r$ coordinate. Only at $r=0$ a singularity is developed.

\section{Bouncing cosmologies}

The existence of wormholes when the cosmological constant is negative motivate us to look for bouncing cosmologies when the cosmological constant is positive. The relevant Einstein metric, $R_{\alpha \beta}=\frac{3}{\ell^{2}} g_{\alpha \beta}$, is now

$$
d s^{2}=-\frac{4 \ell^{4} d t^{2}}{\sigma^{2} f(t)}+g(t)\left(\cos (\theta)^{2} d \phi^{2}+d \theta^{2}\right)+f(t)(d \psi+\sin (\theta) d \phi)^{2} .
$$

with $g(t)=\frac{l^{2}}{\sigma}\left(t^{2}+1\right)$ and

$$
f(t)=\frac{4 \ell^{2}}{\sigma^{2}} \frac{t^{4}+(6-\sigma) t^{2}+\mu t+\sigma-3}{t^{2}+1}
$$

with exactly the same form than (4.3). Therefore, the same analysis applies here regarding regularity. The number of bounces and anti-bounces the spacetime can have is described by figure 1 . The change of coordinates $t=\exp \left(\frac{\tau}{\ell}\right)$, when $\sigma=4$ yields for large $\tau$

$$
d s^{2}=-d \tau^{2}+\frac{l^{2}}{4} \exp \left(\frac{2 \tau}{\ell}\right)\left[\cos (\theta)^{2} d \phi^{2}+d \theta^{2}+(d \psi+\sin (\theta) d \phi)^{2}\right]+O(1)
$$

which is just the Friedmann-Lemaitre-Robertson-Walker metric with spherical topology. There is a straightforward generalization of this cosmology along the lines of the previous section.

\section{Discussion}

In this paper we have constructed the first geometrically non-trivial family of wormhole solutions to four dimensional Einstein gravity with a negative cosmological constant. The 
hypersurfaces perpendicular to the radial coordinate are warped $\mathrm{AdS}_{3}$ spacetimes with the warping that is running along this coordinate. The asymptotic form of the constant- $r$ metric can be either warped or not. The wormhole is traversable and free of closed time like curves. We have generalized the geometry and shown that it is a special limit of the more general charged Plebanski-Demianski spacetime. These spacetimes should now be studied at this new light.

Wormhole geometries in four dimensional, asymptotically AdS spacetimes have received large attention recently due to holography, see for instance [19]. The holographic dual of a highly entangled state of two non-interacting CFTs is an eternal black hole, which has two asymptotically AdS regions that are causally disconnected [20]. It was recently found that the inclusion of an interaction between the two CFTs opens a throat in the bulk which causally connects the boundaries [21], and the size of the throat increases with the rotation in the bulk [22]. Our findings imply that such settings can also take place in vacuum.

When the function $f(r)$ in (4.3) have zeroes, it is possible to cut the spacetime at the first zero and identify the coordinate $u$ to eliminate the conical singularity at the degeneration surface of $\partial_{u}$. This procedure yields a soliton if the zero is of order one. If the zero is of order two then one simply finds another asymptotic region in the bulk spacetime. The interior asymptotic region yields in certain cases an RG flow. The soliton can be thought as a new vacuum of general relativity when the conformal class of the boundary metrics contain (warped) $\mathrm{AdS}_{3}$. These boundary conditions have been used to holographically describe graphene [23].

The introduction of identifications in (2.2) yields warped AdS black holes [11]. It is likely that the same identification in (4.1) yields black holes together with the flow of the warping parameter into the radial direction.

It is worth noting that the "real-world" physical relevance of spacelike warped $\mathrm{AdS}_{3}$ stems from the fact that it arises is the near horizon geometry of the extremal Kerr black hole [24]. This geometry seems to be locally the same than the geometry of our wormhole.

The bouncing cosmology presented in the last section yields a smooth description of the evolution of the Universe. What is remarkable there is that it is possible to recover an standard homogeneous and isotropic Universe for late and early times. Nowadays, the experimental data favours a flat universe. Hence, this cosmological model would only be compatible with the data if the spherical Universe is large enough.

\section{Acknowledgments}

We thank Laura Andrianopoli, Sebastian Bramberger, Elena Caceres, Bianca Cerchiai, Jean-Luc Lehners and Mario Trigiante for discussions. This work was supported in part by the Chilean FONDECYT [Grant No. 1181047, 1170279, 1161418], CONYCT-RCUK [Newton-Picarte Grants DPI20140053 and DPI20140115] and the Alexander von Humboldt foundation. 
Open Access. This article is distributed under the terms of the Creative Commons Attribution License (CC-BY 4.0), which permits any use, distribution and reproduction in any medium, provided the original author(s) and source are credited.

\section{References}

[1] A. Einstein and N. Rosen, The particle problem in the general theory of relativity, Phys. Rev. 48 (1935) 73 [INSPIRE].

[2] M. Visser, Lorentzian wormholes: from Einstein to Hawking, AIP, Woodbury, U.S.A. (1995).

[3] M.S. Morris, K.S. Thorne and U. Yurtsever, Wormholes, time machines and the weak energy condition, Phys. Rev. Lett. 61 (1988) 1446 [INSPIRE].

[4] J.M. Maldacena, The large $N$ limit of superconformal field theories and supergravity, Int. J. Theor. Phys. 38 (1999) 1113 [Adv. Theor. Math. Phys. 2 (1998) 231] [hep-th/9711200] [INSPIRE].

[5] E. Witten and S.-T. Yau, Connectedness of the boundary in the AdS/CFT correspondence, Adv. Theor. Math. Phys. 3 (1999) 1635 [hep-th/9910245] [INSPIRE].

[6] P. Breitenlohner and D.Z. Freedman, Positive energy in Anti-de Sitter backgrounds and gauged extended supergravity, Phys. Lett. 115B (1982) 197 [INSPIRE].

[7] P. Breitenlohner and D.Z. Freedman, Stability in gauged extended supergravity, Annals Phys. 144 (1982) 249 [INSPIRE].

[8] I. Bengtsson and P. Sandin, Anti de Sitter space, squashed and stretched, Class. Quant. Grav. 23 (2006) 971 [gr-qc/0509076] [INSPIRE].

[9] M. Novello and S.E.P. Bergliaffa, Bouncing cosmologies, Phys. Rept. 463 (2008) 127 [arXiv:0802.1634] [INSPIRE].

[10] Y.-F. Cai, D.A. Easson and R. Brandenberger, Towards a nonsingular bouncing cosmology, JCAP 08 (2012) 020 [arXiv: 1206.2382] [INSPIRE].

[11] D. Anninos, W. Li, M. Padi, W. Song and A. Strominger, Warped AdS $S_{3}$ black holes, JHEP 03 (2009) 130 [arXiv:0807.3040] [INSPIRE].

[12] J.M. Maldacena and L. Maoz, Wormholes in AdS, JHEP 02 (2004) 053 [hep-th/0401024] [INSPIRE].

[13] O. Coussaert and M. Henneaux, Selfdual solutions of $(2+1)$ Einstein gravity with a negative cosmological constant, in The black hole: 25 years after, C. Teitelboim ed., World Scientific, Singapore (1998), hep-th/9407181 [INSPIRE].

[14] V. Balasubramanian and P. Kraus, A stress tensor for Anti-de Sitter gravity, Commun. Math. Phys. 208 (1999) 413 [hep-th/9902121] [INSPIRE].

[15] R.B. Mann, Misner string entropy, Phys. Rev. D 60 (1999) 104047 [hep-th/9903229] [INSPIRE].

[16] D.Z. Freedman, K. Pilch, S.S. Pufu and N.P. Warner, Boundary terms and three-point functions: an AdS/CFT puzzle resolved, JHEP 06 (2017) 053 [arXiv:1611.01888] [INSPIRE].

[17] D. Anninos, Sailing from warped $A d S_{3}$ to warped $d S_{3}$ in topologically massive gravity, JHEP 02 (2010) 046 [arXiv:0906.1819] [INSPIRE]. 
[18] J.F. Plebanski and M. Demianski, Rotating, charged and uniformly accelerating mass in general relativity, Annals Phys. 98 (1976) 98 [INSPIRE].

[19] J. Maldacena, A. Milekhin and F. Popov, Traversable wormholes in four dimensions, arXiv: 1807.04726 [INSPIRE].

[20] J.M. Maldacena, Eternal black holes in Anti-de Sitter, JHEP 04 (2003) 021 [hep-th/0106112] [INSPIRE].

[21] P. Gao, D.L. Jafferis and A. Wall, Traversable wormholes via a double trace deformation, JHEP 12 (2017) 151 [arXiv:1608.05687] [INSPIRE].

[22] E. Caceres, A.S. Misobuchi and M.-L. Xiao, Rotating traversable wormholes in AdS, JHEP 12 (2018) 005 [arXiv: 1807.07239] [INSPIRE].

[23] L. Andrianopoli et al., Unconventional supersymmetry at the boundary of $A d S_{4}$ supergravity, JHEP 04 (2018) 007 [arXiv: 1801.08081] [INSPIRE].

[24] J.M. Bardeen and G.T. Horowitz, The extreme Kerr throat geometry: a vacuum analog of $A d S_{2} \times S^{2}$, Phys. Rev. D 60 (1999) 104030 [hep-th/9905099] [INSPIRE]. 